J. Osterburg

Nagoya Math. J.

Vol. 49 (1973), 9-19

\title{
AZUMAYA'S CANONICAL MODULE AND COMPLETIONS OF ALGEBRAS
}

\author{
JAMES OSTERBURG
}

\section{Introduction}

We are concerned with an algebra $S$ over a commutative ring. Precisely $S$ is a non-commutative ring with identity which is also a finitely generated unital $R$ module such that $r(x y)=(r x) y=x(r y)$ for $r$ in $R$ and $x, y \in S$. In section one, we assume $A$ is a commutative, Artinian ring. Following Goro Azumaya (see (1, p. 273)), we define the canonical module $F$ of $A$ to be the injective hull of $A$ modulo the Jacobson radical of $A$ i.e. $F=I(A / J(A))$. Let $S$ be an algebra over $A$, we call a bi-S module $Q$, a canonical $S$ module if $Q$ is isomorphic as a bi-S module to $\operatorname{Hom}_{A}(S, F)$. Azumaya has shown that the canonical bi-S module is uniquely determined, up to isomorphism, by the ring $S$ and is independent of choice of the base ring. In Prop. 1.2 we show that $Q$ as a left $S$ module is the $S$ hull of $S$ modulo $J(S)$. i.e. $Q=I(S / J(S))$. Moreover the left $S$ endomorphism ring of $Q$ is $S$. (See Prop. 1.3.)

In section 2 we consider an algebra $S$ over a commutative ring $R$ (without chain conditions). For any maximal ideal $\mathfrak{p}$ of $R$ let $J(p)$ be the two sided ideal of $S$ such that $\mathfrak{p} S \subset J(\mathfrak{p})$ and $J(p) / p S$ is the Jacobson radical of $S / \mathfrak{p} S$. Then $\bigcap_{p \max R} J(\mathfrak{p})=J(S)$, the Jacobson radical of $S$.

In section 3 we assume $R$ is a commutative, Noetherian ring and $S$ is an $R$ algebra. Let $\mathfrak{p}$ be a maximal ideal of $R$, then Prop. 3.2 states the left $S$ hull of $S / J(\mathfrak{p}), I_{p}$, is $\operatorname{Hom}_{R}(S, I(R / \mathfrak{p}))$.

If we assume $R$ is semilocal, then we show in Prop. 3.4 that $I(S / J(S))$ is countable generated.

In section 4, Prop. 4.1 we show that the left $S$ endomorphism ring of $I_{p}$ is the completion of $S$ with respect to the $p S$-adic topology. Also $I_{p}$ is injective over its endomorphism ring, see Prop. 4.3. If $R$ is semilocal, then the left $S$ endomorphism ring of $I(S / J(S))$ is the completion

Received January 26, 1972. 
of $S$ with respect to the $J(S)$ adic topology. Furthermore, $I(S / J(S))$ is injective over its endomorphism ring, see Propositions 4.2 and 4.4 .

In section 5 , we set $E=\underset{p \max \text { in } R}{\oplus} I_{p}$. We show that the left $S$ endomorphism ring of $E$ is inv. $\lim S / \mathfrak{U}$ where $\mathfrak{H}$ is a left ideal of $S$ such that $S / \mathcal{H}$ is Artinian, see Prop. 5.3. In Prop. 5.5 we show the bicommutator of $E$ is the completion of $S$ with respect to the finite topology.

I want to thank my advisor Goro Azumaya for all of his help and encouragement.

\section{§ 1. The Canonical Module in the Artinian Case}

We assume $A$ is a commutative, Artinian ring and $S$ an algebra over $A$. The Jacobson radical of $S$ (respectively $A$ ) is $J(S)$ (respectively $J(A)$.)

Definition 1.1. The $A$ canonical module is the $A$ injective hull of $A / J(A)$. Denote the canonical module by $F$.

Proposition 1.1. The $A$ canonical module $F$ is a finitely generated $A$ module. The ring map $A \rightarrow \operatorname{End}_{A}(F)$, which sends $a \in A$ to $(x \rightarrow a x)$, $x \in F$ is an isomorphism.

Proof. See Azumaya (1, Prop. 10, p.273)

If $S$ is an algebra over $A$, then $S$ is left and right Artinian.

Definition 1.2. A bi-S module $Q$ is called a canonical $S$-module if $Q$ is isomorphic as a bi-S module to $\operatorname{Hom}_{A}(S, F)$.

Remark 1.1. We regard $\mathrm{Hom}_{A}(S, F)$ as a bi-S module by defining $(s f)=(t \rightarrow f(t s)),(f s)=(t \rightarrow f(s t))$ for $f \in \operatorname{Hom}_{A}(S, F), s, t \in S$.

So with each base ring of $S$, there is a canonical $S$ module. Azumaya has shown that the canonical two sided $S$ module is uniquely determined, up to isomorphism, by the ring $S$ and is independent of the choice of the base ring (see 1, Thm. 21, p. 276).

Proposition 1.2. If $Q$ is the canonical two sided $S$ module, then $Q$ as a left $S$ module (respectively as a right $S$ module) is the left (respectively the right) injective hull of $S / J(S)$ regarding $S / J(S)$ as a left $S$ module (respectively as a right $S$ module). Thus the left (or right) $S$ hull of $S / J$ is a bi-S module. 
Proof. For any base ring $A$ of $S$, as a two sided $S$ module, $Q \simeq$ $\mathrm{Hom}_{A}(S, F)$. Now by (3, Prop. 6.1a, p. 30) $\operatorname{Hom}_{A}(S, F)$ is left and right $S$ injective. It is well known that an injective $S$ module is the hull of its socle. It is also clear that $r_{Q}(J)=\{q \in Q \mid J q=0\}$ is the socle of $Q$. Now $r_{Q}(J)=\operatorname{Hom}_{A}(S / J, F)$ by (1, Lemma 3, p. 275). We decompose $S / J=\bar{S}=\overline{S e}_{1}+\cdots+\overline{S e}_{n}$, where the $\overline{S e}_{i}$ 's are simple subrings and $\bar{e}_{i}$ 's are orthogonal idempotents. Then $r_{Q}(J)=\bigoplus_{i=1}^{n} \operatorname{Hom}_{A}\left(\bar{S} e_{i}, F\right)=\bigoplus_{i=1}^{n} \bar{e}_{i} \bar{S}=$ $S / J$ by (1, Lemma 2, p. 274). Thus the socle of $Q$ as a left (or right $S$ ) module is $S / J$. So as a left (or right $S$ ) module $Q$ is the injective hull of $S / J$.

Proposition 1.3. Let $S$ be an algebra over a commutative, Artinian ring, then the left $S$ injective hull of $S / J, I$, is finitely generated and contains a copy of every simple S-module. Moreover, the map $S$ to End $_{S} I$ which sends $s$ to $(x \rightarrow x s), x \in I, s \in S$ is an isomorphism of rings. We can replace left by right in the above.

Proof. As a bi-S module, $I$ is of $Q F$ type (1, Thm. 19, p. 275). Since $S$ is left and right Artinian, we have established (iii) of Theorem 6 (1, p. 259), which is equivalent to (i) of Theorem $6(1$, p. 259). But (i) Theorem 6 is our result.

\section{§ 2. The Jacobson Radical of an Algebra}

We assume $R$ is an arbitrary commutative ring and $S$ an $R$ algebra.

Proposition 2.1. Let $M$ be a non-zero simple left $S$ module. Then there exists a unique maximal ideal $\mathfrak{p}$ of $R$ such that $\mathfrak{p} M=0$. Thus if $\mathfrak{B}$ is a left maximal ideal of $S$ there exists a unique maximal ideal $\mathfrak{p}$ of $R$ such that $\mathfrak{p} S \subset \mathfrak{B}$. Moreover, $\mathfrak{p}=\left\{r \in R \mid r \cdot 1_{S} \subset \mathfrak{P}\right\}$, if $R \subset$ center of $S$, then $\mathfrak{p}=R \cap \mathfrak{P}$.

Proof. Follows easily from Azumaya (2, Theorem 5, p. 123).

Proposition 2.2. For any algebra $S$ over $R$, let $J(p)$ be, for each maximal ideal $\mathfrak{p}$ of $R$, the two sided ideal of $S$ such that $\mathfrak{p} S \subset J(\mathfrak{p})$ and $J(\mathfrak{p}) / \mathfrak{p} S$ is the Jacobson radical of the residue class algebra $S / \mathfrak{p} S$. Then the radical $J$ of $S$ is the intersection of all the $J(p)$ 's i.e. $J(S)=$ $\bigcap_{p \text { maximal in } R} J(\mathfrak{p})$. So $J(R) \cdot S \subset J(S)$. Moreover, if $\mathfrak{p} \neq \mathfrak{q}$ are maximal ideals of $R$, then $J(\mathfrak{p})+J(\mathfrak{q})=S=\mathfrak{p} S+q S$. 
Proof. The first statement is the corollary of Lemma 2 (2, p. 125). If $\mathfrak{p} \neq \mathfrak{q}$, then $S=R \cdot S=(\mathfrak{p}+\mathfrak{q}) S \subset \mathfrak{p} S+\mathfrak{q} S \subset J(\mathfrak{p})+J(\mathfrak{q}) \subset S$. So $S=$ $\mathfrak{p} S+\mathfrak{q} S=J(\mathfrak{p})+J(\mathfrak{q})$.

§3. From now on we assume $R$ is a commutative, Noetherian ring and $S$ is an $R$ algebra. Thus $S$ is left and right Noetherian. Let $\mathfrak{p}$ be a maximal ideal of $R$.

Remark 3.1. Let $S, R$ and $\mathfrak{p}$ be as above and $i \geq 1$, then $R / \mathfrak{p}^{i}$ is a local, Artinian ring, $S / \mathfrak{p}^{i} S$ is an algebra over $R / \mathfrak{p}^{i}$ and the radical of $S / \mathfrak{p}^{i} S$ is $J(\mathfrak{p}) / \mathfrak{p}^{i} S$.

Proof. Now $S / \mathfrak{p} S$ is finite dimensional over $R / \mathfrak{p}$, so $S / \mathfrak{p} S$ is Artinian. Thus the Jacobson radical is nilpotent i.e. for some $k>0, J(p)^{k} \subset p S$. So $J(\mathfrak{p})^{i k} \subset \mathfrak{p}^{i} S$, but $S / J(\mathfrak{p})$ is semisimple and so has no non-zero nilpotent ideals. Thus $J(\mathfrak{p}) / \mathfrak{p}^{i} S$ is the Jacobson radical of $S / \mathfrak{p}^{i} S$.

Proposition 3.1. Let $\mathfrak{p}$ be a prime ideal of a commutative, Noetherian ring $R$, call the injective hull of $R / \mathfrak{p}, I$, and let $A_{i}=$ $\left\{x \in I \mid p^{i} x=0\right\}$, then $A_{i}$ is a submodule of $I, A_{i} \subset A_{i+1}$ and $I=\bigcup_{i} A_{i}$. Moreover, if $\mathfrak{p}$ is a maximal ideal, then each $A_{i}$ is finitely generated $R$-module, thus $I$ is a countable generated $R$-module.

Proof. See Matlis (4, Theorem 3.4, p. 520) and (4, Theorem 3.11, p. 525).

Proposition 3.2. Let $\mathfrak{p}$ be a maximal ideal of a commutative, Noetherian ring and $S$ an algebra over $R$. Then the left $S$ injective hull of $S / J(\mathfrak{p})$, which we call $I_{\mathfrak{p}}$, is $\operatorname{Hom}_{R}(S, I(R / \mathfrak{p}))$. Thus $I_{\mathfrak{p}}$ becomes in the natural way a bi-S module. Moreover, $\operatorname{Hom}_{R}(S, I(R / p))$ is the union of the canonical $S / \mathfrak{p}^{i} S$ modules i.e. $I_{\mathfrak{p}}=\bigcup_{i} \operatorname{Hom}_{R}\left(S, A_{i}\right)$. We can replace left by right in the above.

Proof. Since $S$ is a finitely generated $R$ module $\operatorname{Hom}_{R}(S, I(R / \mathfrak{p}))=$ $\bigcup_{i} \operatorname{Hom}_{R}\left(S, A_{i}\right)$. Now for each $i>0, \operatorname{Hom}_{R}\left(S, A_{i}\right)=\operatorname{Hom}_{R / p} i\left(S / \mathfrak{p}^{i} S, A_{i}\right)$, let $\bar{S}=S / \mathfrak{p}^{i} S$ and $\bar{R}=R / \mathfrak{p}^{i}$ we observe $\bar{R}$ is commutative, Artinian and $\bar{S}$ is an algebra over $\bar{R}$. By (1, Thm. 17, p. 272) $A_{i}$ is the $\bar{R}$ injective hull of $R / \mathfrak{p}$. Thus for each $i>0, \operatorname{Hom}_{R}\left(S, A_{i}\right)=\operatorname{Hom}_{\bar{R}}\left(\bar{S}, I_{\bar{R}}(R / \mathfrak{p})\right)=Q_{i}$ which is the canonical $\bar{S}$ module. We know by Proposition 1.2 and Remark 3.1, that as a left $\bar{S}$ module $Q_{i}$ is the injective hull of $S / J(\mathfrak{p})$. 
Also $Q_{i} \subseteq Q_{i+1}$, for $A \subset A_{i+1}$, thus $S / J(\mathfrak{p})$ is a large $S$ submodule of $\bigcup_{i} Q_{i}$ $=\operatorname{Hom}_{R}(S, I(R(\mathfrak{p}))$. But Hom $(S, I(R / \mathfrak{p}))$ is injective by (3, Prop. 6.1a, p. 30.). Thus $\operatorname{Hom}_{R}(S, I(R / \mathfrak{p}))$ is the left $S$ injective hull of $S / J(\mathfrak{p})$. For $B$ a subset of $S$, let $r(B)=\left\{y \in I_{\mathfrak{p}} \mid B y=0\right\}$ and $l(B)=\left\{y \in I_{\mathfrak{p}} \mid y B=0\right\}$.

Proposition 3.3. The notation as in Prop. 3.2, then $I_{p}=\bigcup_{i} r\left(p^{i} S\right)=$ $\bigcup_{i} r\left(J(\mathfrak{p})^{i}\right)=\bigcup_{i} l\left(\mathfrak{p}^{i} S\right)=\bigcup_{i} l\left(J(\mathfrak{p})^{i}\right)$.

Proof. Let $i>0$ and regard $Q_{i}$ as an $S$-module, then the $S$ hull of $Q_{i}$ is $I_{\mathfrak{p}}$. Now $r\left(p^{i} S\right)=Q_{i}$ as an $S / p^{i} S$ module (see 1, Cor. Thm. 17, p. 273). So $I_{\mathfrak{p}}=\bigcup_{i} r\left(p^{i} S\right)=\bigcup_{i} l\left(p^{i} S\right)$. Also $S / \mathfrak{p} S$ is Artinian, so for some $k, J(\mathfrak{p})^{k} \subset \mathfrak{p} S . \quad$ Thus $I_{\mathfrak{p}}=\bigcup_{i} r\left(J(\mathfrak{p})^{i}\right)=\bigcup_{i} l\left(J(\mathfrak{p})^{i}\right)$.

We call $R$ semilocal, if $R$ is commutative Noetherian ring with only a finite number of maximal ideals, $\mathfrak{p}_{1}, \cdots, \mathfrak{p}_{t}$.

Proposition 3.4. Let $R$ be a semilocal ring and $S$ an $R$-algebra. Then the left $S$ injective hull of $S / J(S)$ is $\operatorname{Hom}_{R}(S, I(R / J(R))$. Thus $I(S / J(S))$ becomes a bi-S module in the natural way. We can replace left by right in the above.

Proof. By Prop. 2.2 and the Chinese Remainder Theorem, $S / J(S)$ $=S / J\left(\mathfrak{p}_{1}\right) \oplus \cdots \oplus S / J\left(\mathfrak{p}_{t}\right)$, so $I_{S}(S / J(S))=I_{S}\left(S / J\left(\mathfrak{p}_{1}\right)\right) \oplus \cdots \oplus I_{S}\left(S / J\left(\mathfrak{p}_{t}\right)\right)=$ $\operatorname{Hom}_{R}\left(S, I\left(R / \mathfrak{p}_{1}\right)\right) \oplus \cdots \oplus \operatorname{Hom}_{R}\left(S, I\left(R / \mathfrak{p}_{t}\right)\right)=\operatorname{Hom}_{R}(S, I(R / J(R))$.

Let $\mathfrak{P}$ be a left maximal ideal of $S$, we know there exists a unique maximal ideal $p$ of $R$ such that $\mathfrak{p} S \subset \mathfrak{B}$. Moreover, if $R$ is contained in the center of $S$, then $\mathfrak{p}=R \cap \mathfrak{B}$.

Proposition 3.5. Let $\mathfrak{P}$ be a left maximal ideal of an algebra $S$ over a commutative noetherian ring $R$. Call the left $S$ injective hull of $S / \mathfrak{R}, I$. Let $r\left(\mathfrak{p}^{i} S\right)$ be $\left\{x \in I \mid\left(\mathfrak{p}^{i} S\right) x=0\right\}$. Then $I=\bigcup_{i} r\left(\mathfrak{p}^{i} s\right)=\bigcup_{i} r\left(J(\mathfrak{p})^{i}\right)$.

Proof. Since $S / \mathfrak{R}$ is a simple left $S$ module, it is a simple left $S / J(\mathfrak{p})$ module. Also $S / J(\mathfrak{p})$ is completely reducible, so $S / \mathfrak{P}$ is isomorphic to a direct summand of $S / J(\mathfrak{p})$. Thus $I$ is a direct summand of $I_{\mathfrak{p}}=$ $\bigcup_{i} r\left(\mathfrak{p}^{i} S\right)$. So $I=\bigcup_{i} r_{I}\left(\mathfrak{p}^{i} S\right)$.

Proposition 3.6. Let $R, \mathfrak{p}, S$ and $\mathfrak{\beta}$ be as above. Then the left $S$ injective hull of $S / \mathfrak{P}$ and $S / J(\mathfrak{p})$ are countable generated. 
Proof. Propositions 3.3, 3.5 and 1.3.

Proposition 3.7. If $R$ is a semilocal ring, then the left (or right) $S$ injective hull of $S / J(S)$ is countable generated.

Proof. Propositions 3.6 and 3.4.

$\S$ 4. We fix a maximal ideal $p$ of a commutative, Noetherian ring $R$. Let $S$ be an $R$-algebra with the "p $S$-adic" topology. We define the completion of $S$ with respect to the $p S$-adic topology to be inv. $\lim S / p^{i} S$, denoted by $\hat{S}_{\mathfrak{p}}$. Now $I_{\mathfrak{p}}$ is a right $\hat{S}_{\mathfrak{p}}$ module. For let $\hat{s}=\left(s_{i}+\mathfrak{p}^{i} S\right) \in \hat{S}_{\mathfrak{p}}$ and $x \in I_{\mathfrak{p}}$. Then for $k>0, x\left(\mathfrak{p}^{k} S\right)=0$, (by Prop. 3.3) define $x \hat{s}=x s_{k}$. If $x\left(\mathfrak{p}^{j} S\right)=0$, assume $j<k$, then $s_{k}-s_{j} \in \mathfrak{p}^{j} S$ so $x\left(s_{k}-s_{j}\right)=0$ or $x s_{k}=$ $x s_{j}$. Since $I_{\mathfrak{p}}$ is a bi $S$-module (Prop. 3.2), $I_{\mathfrak{p}}$ becomes a bi-S $-\hat{S}_{\mathfrak{p}}$ module.

We also consider $S$ with the $J(\mathfrak{p})$-adic topology. We call inv. $\lim S / J(\mathfrak{p})^{i}$, the completion of $S$ with respect to the $J(p)$-adic topology, denoted by $\hat{S}_{J(\mathfrak{p})}$. As above, $I_{\mathfrak{p}}$ becomes a bi-S $-\hat{S}_{J(\mathfrak{p})}$ module. Since $\mathfrak{p} S \subset J(\mathfrak{p})$ and $J(\mathfrak{p})^{k} \subset \mathfrak{p} S$, then $\hat{S}_{\mathfrak{p}}=\hat{S}_{J(\mathfrak{p})}$.

Proposition 4.1. The $S$ endomorphism ring of $I_{\mathfrak{p}}$ (as either a left or right $S$ module) is the completion of $S$ with respect to the pS-adic or $J(\mathfrak{p})$-adic topologies i.e. $\operatorname{End}_{S} I_{\mathfrak{p}}=\hat{S}_{\mathfrak{p}}$.

Proof. Since $\left(\bigcap_{i} \mathfrak{p}^{i} S\right) \cdot I_{\mathfrak{p}}=0, I_{\mathfrak{p}}$ is a left $S / \bigcap_{i} \mathfrak{p}^{i} S$ module. In other words, we may assume $S$ is Hausdorff in the $p S$-adic topology. Now

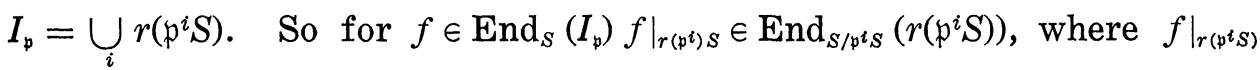
means $f$ restricted to $r\left(\mathfrak{p}^{i} S\right)$. It follows that $\operatorname{End}_{S} I_{\mathfrak{p}}=\operatorname{inv}$. $\lim \operatorname{End}_{S / \mathfrak{p} i S}$ $\left(r\left(\mathfrak{p}^{i} S\right)\right)$. We now find for each $i>0, \operatorname{End}_{S / \mathfrak{p} i S}\left(r\left(\mathfrak{p}^{i} S\right)\right)$.

In the proof of Prop. 3.3, we showed $r\left(p^{i} S\right)$ as a left $S / p^{i} S$ module is the $S / \mathfrak{p}^{i} S$ hull of $S / J(\mathfrak{p})$. Using Prop. 1.3, we conclude $\operatorname{End}_{S / \mathfrak{p}^{i} S}\left(r\left(\mathfrak{p}^{i} S\right)\right)$ $=S / \mathfrak{p}^{i} S$, the isomorphism given by right multiplication. Since the following diagram commutes

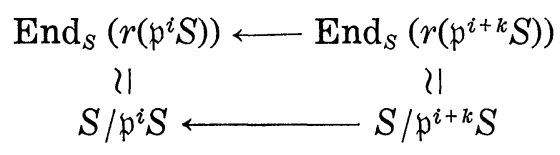

we conclude that $\operatorname{End}_{S}\left(I_{\mathfrak{p}}\right)=$ inv. $\lim \operatorname{End}_{S / \mathfrak{p} i S}\left(r\left(\mathfrak{p}^{i} S\right)\right)=\operatorname{inv} . \lim S / \mathfrak{p}^{i} S$.

By a semilocal ring $R$, we mean a commutative, Noetherian ring with only a finite number of maximal ideals, $\mathfrak{p}_{1}, \cdots, \mathfrak{p}_{t}$. 
Proposition 4.2. Let $R$ be a semilocal ring and $S$ an algebra over $R$. Then the endomorphism ring of the injective hull of $S / J(S), I(S / J(S))$, is the completion of $S$ with respect to the $J(S)$-adic topology.

Proof. We have seen (Prop. 3.4) $I(S / J(S))=\bigoplus_{i=1}^{t} I\left(S / J\left(p^{i}\right)\right)$. Let $\mathfrak{p} \neq \mathfrak{q}$ be maximal ideals of $R$, we show for $f \in \operatorname{Hom}_{S}\left(I_{p}, I_{q}\right)$, then $f=0$. Let $x \in I_{\mathfrak{p}}$, then $\left(\mathfrak{p}^{k} S\right) x=0$ and $\left(\mathfrak{q}^{l} S\right) f(x)=0$ for $k, l>0$, by Prop. 3.3. Since $\mathfrak{p}^{k}+\mathfrak{q}^{l}=R$, there exists $a \in \mathfrak{p}^{k}, b \in \mathfrak{q}^{l}$ such that $a+b=1$. So $f(x)=$ $f(a x+b x)=f(a x)+b f(x)=0$. Thus $f \equiv 0$. We conclude $\operatorname{End}_{S}(I(S / J(S))$ $=\bigoplus_{i=1}^{t} \operatorname{End}_{S}\left(I_{p_{i}}\right)=\bigoplus_{i=1}^{t}$ inv. $\lim S / \mathfrak{p}_{i}^{j} S=S \underset{R}{\bigotimes_{R}}\left(\bigoplus_{i=1}^{t}\right.$ inv. $\left.\lim R / \mathfrak{p}_{i}^{j}\right)=S \underset{R}{\otimes}$ inv. $\lim$ $R / J(R)^{i}=\operatorname{inv} \cdot \lim S / J(R)^{i} S$.

Now $S / J(R) \cdot S$ is an algebra over the commutative, Artinian ring $R / J(R)$. So $S / J(R) S$ is Artinian, thus its Jacobson radical is nilpotent of index $k$, so $J(S)^{k} \subset J(R) S$. Also $J(R) S \subset J(S)$, thus inv. $\lim S / J(R)^{k} S=$ inv. $\lim S / J(S)^{k}$.

Returning to a commutative, Noetherian ring $R, \mathfrak{p}$ a maximal ideal of $R$ and $S$ an $R$ algebra, we call the left $S$ endomorphism ring of $I_{p}, H_{p}$. We have seen (Prop. 4.1) that $H_{p}$ is $\hat{S}$, the completion of $S$ with respect to the $J(\mathfrak{p})$-adic topology. Let $\widehat{J(\mathfrak{p})}=\operatorname{inv} \cdot \lim J(\mathfrak{p}) / J(\mathfrak{p})^{i}$, then $\hat{S_{\mathfrak{p}}} / \widehat{J(\mathfrak{p})}$ is $S / J(p)$ as left $S$ modules.

Proposition 4.3. The notation as above, then $I_{\mathfrak{p}}$ is an injective $H_{\mathfrak{p}}$ module. In fact, $I_{\mathfrak{p}}$ is the $H_{\mathfrak{p}}$ injective hull of $\hat{S}_{\mathfrak{p}} / \widehat{J(\mathfrak{p})}$. Moreover, $\hat{A}_{k}=$ $\left\{x \in I_{\mathfrak{p}} \mid x \widehat{J(\mathfrak{p})^{k}}=0\right\}$ and $A_{k}=\left\{x \in I_{\mathfrak{p}} \mid x J(\mathfrak{p})^{k}=0\right\}$ are equal for all $k>0$.

Proof. Denote the right $\hat{S}$ module $\hat{S} / \widehat{J(p)}$ by $C$. Let $D$ be the right $\hat{S}$ hull of $C$. We show $C$ is an essential $S$ submodule of $D$. Now $\hat{S}$ is a left and right Noetherian ring, since it is an algebra over inv. $\lim R / \mathfrak{p}^{i}$. So $D=\bigcup_{i} D_{i}$, where $D_{i}=\left\{x \in D \mid x \widehat{J(\mathfrak{p})^{i}}=0\right\}$. Let $0 \neq d \in D$ so $d \in \hat{A}_{k}$ for some $k$. Also there exists $\hat{s}=\left(s_{i}+J(\mathfrak{p})^{i}\right) \in \hat{S}_{\mathfrak{p}}$ such that $0 \neq d \hat{s} \in C$; hence $0 \neq d s_{k} \in C$. So $C$ is an essential right $S$ module of $D$. Also by Prop. 3.2, $I_{\mathfrak{p}}$ is a right $S$ injective module.

Thus we can find a right $S$ map $h$ such that $h g=i$, where $g=$ $(S / J(p) \simeq \hat{S} / \widehat{J(p)} \subseteq D)$ and $i$ are viewed as right $S$ maps. 


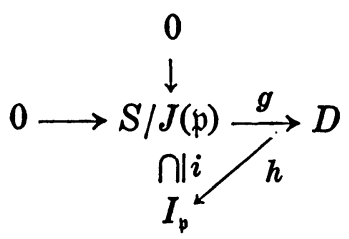

Now $h$ is one to one for $S / J(p)$ is an essential right $S$ module. Since $I_{p}$ is a right $\hat{S}$ module and $D$ is an injective $\hat{S}_{\mathfrak{p}}$ module, $D$ is a direct summand of $I_{p}$. However, $i$ is essential, so $D=I_{p}$. The equality of $\hat{A}_{k}$ and $A_{k}$ follows from $\widehat{J(p)}=J(\mathfrak{p}) \hat{S}$.

§5. As usual we assume $R$ is commutative Noetherian and $S$ is an $R$-algebra. The direct sum (as left $S$ modules) of the $I_{\mathfrak{p}}$ 's, $\mathfrak{p}$ ranging over all maximal ideals of $R$, we call the canonical cogenerator, $E$. i.e. $E=\oplus I_{p}$. Now $E$ is the left $S$ hull of $F$, where $F$ is the direct sum of the $S / J(\mathfrak{p})$ 's. Moreover, since $S$ is a finitely generated $R$-module, $E=$ $\operatorname{Hom}_{R}\left(S, \underset{p \max i n}{\oplus} I_{R}(R / p)\right)$. Thus $E$ becomes in the natural way a bi-S module and the right $S$ hull of $F$. Because $E$ contains a copy of each simple left (right) $S$ module, $E$ is left (right) $S$ cogenerator; hence, $E$ is faithful as a left (right) $S$ module.

We denote by $\boldsymbol{P}$ the totality of all products of powers of maximal ideal of $R$. If $\mathfrak{p}_{1}^{t_{1}} \cdots \mathfrak{p}_{m}{ }^{t_{n}} \in \boldsymbol{P}$, then $\mathfrak{p}_{1}^{t_{1}} \cap \cdots \cap \mathfrak{p}_{m}{ }^{t_{n}}=\mathfrak{p}_{1}{ }^{t_{1}} \cdots \mathfrak{p}_{n}{ }^{t_{n}}$.

For $B$ a subset of $S$, we call $r(B)=\{x \in E \mid B x=0\}$ and $l(B)=$ $\{x \in E \mid x B=0\}$.

Proposition 5.1. $\quad E=\bigcup_{w \in P} r(w S)=\bigcup_{w \in \boldsymbol{P}} l(w s)$

Proof. Let $x \in E$, then $x=x_{1}+\cdots+x_{n}, x_{i} \in I_{p_{i}} ; i=1, \cdots, n$. Вy Proposition 3.3, $\left(\mathfrak{p}_{1}{ }^{{ }_{1}} S\right) x_{1}=0 ; \cdots ;\left(\mathfrak{p}_{n}{ }^{k_{n}} S\right) x_{n}=0$. So $\mathfrak{p}_{1}{ }^{k_{1}} \cdots \mathfrak{p}_{n}{ }^{k_{n}}=w \in \boldsymbol{P}$ and $(w S) x=0$.

The $n$-adic topology of $S$ has as a basis of neighborhoods of zero ideals of the form $w S, w \in \boldsymbol{P}$. We partially order $\boldsymbol{P}$ by inclusion. In fact, $\boldsymbol{P}$ is a direct set. We call $S^{*}=\underset{w \in \boldsymbol{P}}{\operatorname{inv}} \lim S / w S$, the completion of $S$ with respect to the $n$-adic topology. Furthermore, $E$ is a bi-S $-S^{*}$ module. Let $s^{*}=\left(s_{w}+w S\right) \in S^{*}, s_{w} \in S, w \in \boldsymbol{P}$ and $x \in E$, then $0=x(v S)$ for $v \in \boldsymbol{P}$, define $x s^{*}=x s_{v}$. If $x(w S)=0$ for $w \in \boldsymbol{P}$, then $x((v w) S)=0$. Thus $s_{v}-s_{v w} \in v S$ and $s_{w}-s_{v w} \in w S$, so $x s_{v}=x s_{v w}=x s_{w}$. We conclude the multiplication is well defined. 
For any $B \subset S$, let $l_{F}(B)=\{x \in F \mid B x=0\}$ and $l_{E}(B)=\{x \in E \mid B x=$ $0\}, l_{F}(B) \subset l_{E}(B)$. For a fixed $w \in P$, let $\bar{S}=S / w S$ and $\bar{R}=R / w, \bar{S}$ is an algebra over the commutative, Artinian ring $\bar{R}$. Thus $\bar{S}$ is both left and right Artinian.

Proposition 5.2. The notation as above. If $Q=r_{E}(w S)$, then $Q$ is

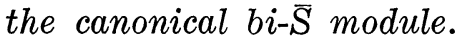

Proof. Since $E$ is the left $S$ hull of $F, r_{E}(w S)$ is the left $\bar{S}$ hull of $r_{F}(w S)$. (See 1, Thm. 17, p. 272). Now let $w=\mathfrak{p}_{1}{ }^{k_{1}} \cdots \mathfrak{p}_{t}{ }^{k_{t}}, \mathfrak{p}_{1}, \cdots, \mathfrak{p}_{t}$ maximal ideals of $R$. We show $r_{F}(w S)=S / J\left(\mathfrak{p}_{1}\right) \oplus \cdots \oplus S / J\left(\mathfrak{p}_{t}\right)$. Since $\mathfrak{p}_{1} S \subset J\left(\mathfrak{p}_{1}\right), \cdots, \mathfrak{p}_{t} S \subset J\left(\mathfrak{p}_{t}\right)$, we have $r_{F}(w S) \supseteq S / J\left(\mathfrak{p}_{1}\right) \oplus \cdots \oplus S / J\left(\mathfrak{p}_{t}\right)$. Let $x \in r_{F}(w S)$, so $x=\bar{x}_{1}+\cdots+\bar{x}_{n}, 0 \neq \bar{x}_{i}=x_{i}+J\left(\mathfrak{q}_{i}\right)$, for $x_{i} \in S$ and $\mathfrak{q}_{i}$ a maximal ideal of $R$ for $i=1, \cdots, n$. Now $(w S) x=0$ implies $(w S) x_{1} \subset$ $J\left(\mathfrak{q}_{1}\right), \cdots,(w S) x_{n} \subset J\left(\mathfrak{q}_{n}\right)$. If $\mathfrak{q}_{1} \neq \mathfrak{p}_{1}, \cdots, \mathfrak{p}_{t}$, then $\mathfrak{q}_{1}+w=R$. Thus $x_{1} \in$ $x_{1}\left(\mathfrak{q}_{1}+w\right) S \subset x_{1}\left(\mathfrak{q}_{1} S\right)+x_{1}(w S) \subset J\left(\mathfrak{q}_{1}\right)$ or $\bar{x}_{1}=0$. However, we assumed $\bar{x}_{1} \neq 0$, thus $\mathfrak{p}_{1}=\mathfrak{q}_{1}$ (after renumbering) continuing we see $\mathfrak{q}_{i}=\mathfrak{p}_{i}$ (after renumbering) and $t \geqq n$. Thus $r_{F}(w S)=S / J\left(\mathfrak{p}_{1}\right) \oplus \cdots \oplus S / J\left(\mathfrak{p}_{t}\right)$ so $r_{E}(w S)$ $=I_{\bar{S}}\left(r_{F}(w S)\right)=I_{\bar{S}}\left(S / J\left(\mathfrak{p}_{1}\right) \oplus \cdots \oplus S / J\left(\mathfrak{p}_{t}\right)\right)=I_{\bar{S}}(\bar{S} / J(\bar{S}))=\operatorname{Hom}_{\bar{R}}\left(\bar{S}, I_{\bar{R}}(\bar{R} / J(\bar{R}))\right.$ by Prop. 1.2. Thus $r_{E}(w S)$ as a bi- $\bar{S}$ module is the canonical $\bar{S}$ module.

Proposition 5.3. The endomorphism ring of $E$ is the completion of $S$ with respect to the $n$-adic topology.

Proof. Since $E=\bigcup_{w \in P} r(w S)$ (Prop. 6.1) $\operatorname{End}_{S} E=\underset{w \in \boldsymbol{P}}{\text { inv. }} \lim _{S / w S}$ $\left(r_{E}(w S)\right)$. By Propositions 5.2, 1.2 and $1.3 S / w S=$ End $\left(r_{E}(w S)\right)$ by $(a+w S) \rightarrow(x \rightarrow x s), a \in S, x \in r(w S)$. If $w S \subset v S$, then the following diagram commutes

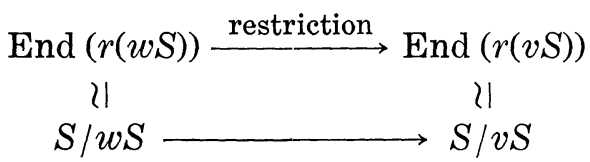

So $\operatorname{End}_{S}(E)=\underset{w \in P}{\text { inv. }} \lim S / w S$.

The question arises: is $E$ injective over its endomorphism ring? F. L. Sandomierski has shown that as long as $E$ has an infinite number of direct summands, then $E$ is not injective over its endomorphism ring. (See Sandomierski) (5, Thm. 1, p. 244).

Let $U$ be the collection $\{U\}$ of left ideals of $S$ such that $S / U$ is left 
Artinian. We order $\boldsymbol{U}$ by inclusion; since the intersection of two ideals of $\boldsymbol{U}$ is in $\boldsymbol{U}, \boldsymbol{U}$ is directed. We call the $\underset{v \in \boldsymbol{U}}{\operatorname{inv}} \lim S / U$ the completion of $S$ with respect to $U$ topology. Now $S / U$ has a composition series $S / U=M_{0} \supset M_{1} \supset \cdots \supset M_{n}=0$ for $U \in U$.

By Prop. 2.2 there exists a unique maximal $p_{i}$ of $R$ such that $p_{i} M_{i} \subset M_{i+1}$ for $i=0, \cdots, n-1$. Now $p_{n-1} \cdots p_{0}(S / U)=0$ i.e. if $w=$ $p_{n-1} \cdots p_{0}$, then $w S \subset U$ and $w \in \boldsymbol{P}$. Furthermore, by the Jordan-Hölder Theorem $w$ is unique. Thus we show for each $U \in U$ there exists a $w \in P$ such that $w S \subset U$ i.e. $\{w S \mid w \in P\}$ is cofinal in $\boldsymbol{U}$.

Proposition 5.4. The endomorphism ring of $E$ (as a left $S$ module) is the completion of $S$ with respect to the $\boldsymbol{U}$ topology.

Proof. We have seen $\{w S \mid w \in \boldsymbol{P}\}$ is cofinal in $\boldsymbol{U}$. Thus $\operatorname{End}_{S} E=$ $\underset{w \in \boldsymbol{P}}{\operatorname{inv}} \lim S / w S=$ inv. $\lim _{\boldsymbol{U} \in \boldsymbol{U}} S / U$.

The finite topology on $S$ has basic neighborhoods of zero of the form $U_{x_{1} \ldots x_{n}}(0)=\left\{s \in S \mid s x_{1}=\cdots=s x_{n}=0\right\}$ for $x_{1}, \cdots, x_{n} \in E$. Since $E$ is faithful the finite topology is Hausdorff. Moreover, by an argument similar to the proof of Prop. 5.4 for each $U_{x_{1} \cdots x_{n}}(0), x_{1}, \cdots, x_{n} \in E$ there exists a $w \in \boldsymbol{P}$ such that $w S \subset U_{x_{1} \cdots x_{n}}(0)$. Thus the finite topology is coarser than the $n$-adic topology and the $n$-adic topology is Hausdorff.

By the bicommutator of $E$ (Bic $(E)$ ) we mean the set of all endomorphisms of $E$ as an Abelian group which commutes with every element of $H\left(=\operatorname{End}_{S} E\right)$.

Proposition 5.5. The bicommutator of $E$ is the completion of $S$ with respect to the finite topology.

Proof. Let $x_{1}, \cdots, x_{n} \in E$ and $U=U_{x_{1} \cdots x_{n}}(0)$, we have a $w \in \boldsymbol{P}$ such that $w S \subseteq U$. So $S / U$ can be regarded as a module over an artinian ring $S / w S$. We define a product on $S / U \times\left(x_{1} H+\cdots+x_{n} H\right) \rightarrow E$, by $\left(s+U, \sum^{n} x_{i} h_{i}\right) \rightarrow \sum_{i=1}^{n} s x_{i} h_{i} \in E$. It is easy to see that $S / U$ and $x_{1} H$ $+\cdots+x_{n} H$ form an orthogonal pair with respect to $E$. See (1, p. 254). Now $E$ is a quasi-Frobenius bi-S $-H$ module because $E$ is left $S$ injective and contains a copy of every simple left $S$ module (See (1, Thm. 4, p. 257)). Furthermore $S / U$ has a composition series as a left $S / w S$ module; hence, $S / U$ has a composition series as a left $S$ module for $w S \subseteq U$. Thus by (1, Prop. 2, p. 254) $x_{1} H+\cdots+x_{n} H$ has a composition series 
as a right $H$ module and $S / U=\operatorname{Hom}_{S}\left(x_{1} H+\cdots+x_{n} H, E\right)$ by $(s+U)$ $\left.\rightarrow\left(\sum x_{i} h_{i} \rightarrow \sum s x_{i} h_{i}\right)\right)$. If $x_{1} H_{1}+\cdots+x_{n} H \subseteq y_{1} H+\cdots+y_{t} H, x_{1}, \cdots$, $x_{n}, y_{1}, \cdots, y_{t} \in E$, then $U_{x_{1} \cdots x_{n}}(0) \supseteq U_{y_{1} \cdots y_{t}}(0)$. The following diagram commutes

$$
\begin{gathered}
S / U_{y_{1} \cdots y_{t}}(0) \longrightarrow S / U_{x_{1} \cdots x_{n}}(0) \\
\operatorname{Hom}_{h}\left(y_{1} H+\cdots+y_{t} H, E\right) \longrightarrow \operatorname{Hom}_{H}\left(x_{1} H+\cdots+x_{n} H, E\right)
\end{gathered}
$$

Thus

$$
\text { inv. } \begin{aligned}
\lim S / U_{y_{1} \cdots y_{n}}(0) & =\text { inv. } \lim \operatorname{Hom}_{H}\left(y_{1} H+\cdots+y_{t} H, E\right) \\
& =\operatorname{Hom}_{I H}\left(\operatorname{dir} \lim y_{1} H+\cdots+y_{n} H, E\right) \\
& =\operatorname{Hom}_{H}(E, E) .
\end{aligned}
$$

Proposition 5.6. If $R$ is a commutative, Noetherian ring, then the completion of $R$ with respect to the n-adic topology equals the completion of $R$ with respect to the finite topology.

\section{BIBLIOGRAPHY}

1) Azumaya, Goro, "A Duality Theory for Injective Modules (Theory for Quasi Frobenius Modules)", American J. of Math. 81 (1959), pp. 249-278.

2) Azumaya, Goro, "On Maximally Central Algebras", Nagoya Math. J., 2 (1951), pp. 119-150.

3) Cartan, H. and S. Eilenberg, Homological Algebra, Princeton, N. J.: Princeton University Press, 1956.

4) Matlis, Eben, "Injective Modules over Noetherian Rings", Pacific J. of Math., 8 (1958), pp. 511-528.

5) Sandomierski, F. L., "Some Examples of Right Self Injective Rings which are not left Self Injective”, P.A.M.S., 26 (1970), pp. 244-245.

University of Cincinnati

Taft Fellow and Indiana University 